\title{
Assessment of Immunogenicity of CP8, CLFA-FnBPB and CP8-CLFA-FnBPB Antigens
}

\author{
${ }^{1,2}$ Xiuli Liu, ${ }^{1}$ Yongqing Hao, ${ }^{1}$ Shuyi Wang, \\ ${ }^{1}$ Airong Zhang, ${ }^{3}$ Yuxi Wang, ${ }^{1}$ Yu Guo and ${ }^{1}$ Chunguang Xu \\ ${ }^{1}$ Faculty of Veterinary Medicine, Inner Mongolia Agricultural University, Hohhot, 010020, China \\ ${ }^{2}$ Veterinary Research Institute, Inner Mongolia Academy of Agricultural \& Animal Husbandy Sciences, Hohhot, 010031, China \\ ${ }^{3}$ Lethbridge Research Centre, Agriculture and Agri-Food Canada, P.O. Box 3000, Lethbridge, AB, T1J 4B1, Canada
}

Received 2013-07-12, Revised 2013-07-12; Accepted 2013-08-27

\begin{abstract}
To assess the effects of conjugating Capsular Polysaccharide (CP) with Clumping factor A (ClfA) and Fibronectin-Binding Proteins B (FnBPB) on the immunogenicity of the antigen, CP was isolated and purified from serotype 8 (CP8) Staphylococcus aureus from bovine mastitis dairy herds in Inner Mongolia and ClfA-FnBPB was produced with Escherichia coli encoding a fusion protein ClfA-FnBPB gene. The CP8-ClfA-FnBPB complete antigen was constructed by first attaching Alcohol Dehydrogenase (ADH) to CP8 which was then combined with ClfA-FnBPB at the presence of EDC (1-ethyl-3, (3-dimethylaminopropyl carbodiimide). The immunogenicity of CP8, ClfA-FnBPB and CP8- ClfA-FnBPB was determined by intraperitoneal injecting 408 -wk old of Balb/c mice four groups with $0.2 \mathrm{ml} / \mathrm{mouse}$ of $0.9 \%$ $\mathrm{NaCl}$ (Control) or CP8, ClfA-FnBPB and CP8-ClfA-FnBPB solution. The mice were group-fed with same pellet diet in an environmentally controlled room with a constant temperature of $22^{\circ} \mathrm{C}$ and $12 \mathrm{~h} / 24 \mathrm{~h}$ light. The concentration of CP8, ClfA-FnBPB or CP-ClfA-FnBPB in respective solutions was 50, 25 or $75 \mu \mathrm{g}$ $\mathrm{mL}^{-1}$. Two injections were given, on $\mathrm{d} 7$ and $\mathrm{d} 21$ of the experiment. Blood samples were taken on $\mathrm{d} 0, \mathrm{~d} 14$, $\mathrm{d} 21$ and $\mathrm{d} 28$ and measured for the antibodies titer. On d28, all mice were injected with CP8 type of $S$. aureus and the mortality of the mice determined for the following 14 days (challenge test). The results showed that ClfA-FnBPB was successfully expressed by $E$ coli and the isolated and purified ClfA-FnBPB was successfully conjugated with CP8 to yield a CP8-ClfA-FnBPB complete antigen. Mice immunized with CP8ClfA-FnBPB had higher antibody titer than that of CP8 $(p<0.01)$ and ClfA-FnBPB $(p<0.05)$ groups. Antibody titer in mice immunized with ClfA-FnBPB antigen was also higher $(\mathrm{p}<0.05)$ than that of CP8 and $0.9 \% \mathrm{NaCl}$ groups. Survival rate of the mice at the end of 2-wk challenge test for Control, CP8, ClfA-FnBPB and CP8-ClfAFnBPB groups were 2030,60 and $80 \%$ respectively. These results demonstrated that conjugating CP8 with ClfA-FnBPB to form CP8-ClfA-FnBPB complete antigen significantly increased immunogenicity of the antigen and markedly enhanced the survival rate of the mice infected with the bacteria. The information suggests that vaccine based on the CP8-ClfA-FnBPB antigen would be more effective in controlling bovine mastitis than those based on CP8 and ClfA-FnBPB alone, but further animal study is needed.
\end{abstract}

Keywords: S. Aureus, Capsular Polysaccharide, CP8-ClfA-FnBPB, Immunogenicity Mice

\section{INTRODUCTION}

Mastitis caused by the infection of Staphylococcus aureus occurs in dairy herds globally and is a serious disease threatening dairy industry in Inner Mongolia, a major dairy production region of China (Siqinmenghe, 2012). It has been regarded that the primary virulent factor in $S$. aureus infection is extracellular Capsule Polysaccharide (CP) serotype (Wu and Huang, 2003). Eleven serotypes of CP (CP1 to CP11) have been Corresponding Author: Yongqing Hao, Faculty of Veterinary Medicine, Inner Mongolia Agricultural University, Hohhot, 010020, China 
defined so far (Zhang and Wang, 2003), which exist in more than $90 \%$ of S.aureus strains. Among them, CP8 is the most prevalent serotype globally (Creuzenet and Joseph, 2001; Lin et al., 2005; Zhang et al., 2004). Our previous study showed that CP8 strains accounted $52.6 \%$ of high pathogenic S.aureus strains isolated from bovine mastitis in Inner Mongolia of China and the rest (47.4\%) were other types and/or nontyped strains (Guo et al., 2011). The other factors determining the virulence of $S$. aureus are adhesins such as Staphylococcal Protein A (SPA), Fibronectin-Binding Proteins A (FnBPA) and B (FnBPB) and Clumping factor $\mathrm{A}(\mathrm{ClfA})$ and $\mathrm{B}(\mathrm{ClfB})$ proteins $(\mathrm{Ni}$ Eidhin et al., 1998; Visai et al., 2006; Wann et al., 2000) that play a crucial role in bacterial adhension and colonization to breast tissue (Stutz et al., 2011). Both CP and adhensins have individually been considered as potential antigen candidates for developing vaccine against $S$. aureus (Daum and Spellberg, 2012). However, the traditional vaccine available for mastitis (Chang et al., 2008; Middleton, 2008) is rather ineffective due to the fact that animal is lack of immune response to new infections (Reid and Szymanski, 2010). It has been suggested that vaccine produced from the combinations of antigens including CPs and adhensins would be an effective way against bovine mastitis caused by $S$. aureus (Lorena et al., 2008; Josefsson et al., 2001), but little information is available in this area.

The objectives of this study were to assess the immunogenicity of CP8, ClfA-FnBPB and CP8-ClfAFnBPB antigens and to determine the efficacy of the antibody produced from these antigens against $S$. aureus.

\section{MATERIALS AND METHODS}

Monoclonal antibody of CP8 was produced from S.aureus anti-CP8 MAb 8E8 (Lot\#237.118) by Dr. Tessie McNeely of Merck \& Co., Inc; Rahway, NJ. US. Alcohol Dehydrogenase (ADH), 1-ethyl-3, (3-dimethylaminopropyl carbodiimide, EDC), NSuccinimidyl N-methylcarbamate (sulfo-NHS), DNase I, RNase A, Lysostaphin and Proteinase $\mathrm{K}$ were obtained from Sigma (St Louse, MS, US) and Sephacryls-300 was obtained from Duly Shanghai (Shanghai, China).

Isopropyl- $\beta$-d-thiogalactoside (IPTG) was obtained from TaKaRa (Dalian, China).

\subsection{Construction of CP8-CIfA-FnBPB Complete Antigen \\ 2.1.1. Extraction and Purification of CP8}

The isolation and identification of CP8 serotype of $S$. aureus strains were reported by Guo et al. (2011). The extraction of CP8 was carried out using a method described by Fournier et al. (1984) and James et al. (2009) with some modifications. Briefly, the identified CP8 strain of $S$. aureus as described in Guo et al. (2011) was first cultured at $37^{\circ} \mathrm{C}$ on 200 Columbia blood agar plates for $24 \mathrm{~h}$. Colonies of the bacteria in each plate were then suspended into $5 \mathrm{~mL}$ of phosphate buffer (PB, $10 \mathrm{mM} ; \mathrm{pH}=7.4$ ) with scratching and suspensions from all plates were combined which was subsequently autoclaved at $121^{\circ} \mathrm{C}$ for $2 \mathrm{~h}$. The autoclaved cell suspension was centrifuged $(3,500 \times \mathrm{g}, 30 \mathrm{~min})$ and the supernatant $(\sim 400 \mathrm{~mL})$ was added DNase $(0.5 \mathrm{mg}$ $\left.\mathrm{mL}^{-1}\right)$, RNase A $\left(25 \mu \mathrm{g} \mathrm{mL}^{-1}\right)$, lysostaphin $(0.25 \mathrm{mg}$ $\left.\mathrm{mL}^{-1}\right)$ and proteinase $\mathrm{K}\left(1.25 \mathrm{mg} \mathrm{mL} \mathrm{mL}^{-1}\right)$. The mixture was stored at room temperature for $24 \mathrm{~h}$, filtered through a $0.22-\mu \mathrm{m}$ filter membrane (PES, $\varphi 40$, Haining Ltd., Zhejiang, China) thereafter and the filtrate was freezedried. The dried powder of CP8 crude extract was then dissolved into PB solution and purified by sephacryls300 with method described by James et al. (2009), lyophilized and the dried powder was stored in sealed container at $-20^{\circ} \mathrm{C}$. The $\mathrm{CP} 8$ content of the purified extract was determined by phenol-sulfuric acid method Wang (2008) and the contents of protein, dsDNA, ssDNA and RNA were determined using a Nucleic Acid Protein Instrument (AG 22331, Eppendorf Hamburg).

\subsection{Induction, Expression and Purification of CIfA-FnBPB}

The induction and expression of ClfA-FnBPB was conducted with Escherichia coli encoding a fusion protein ClfA-FnBPB gene that was constructed in this laboratory (Fan, 2011). E. coil (BL21) was firstly precultured in Liquid Broth (LB) medium for three generations. The overnight-grown third generation of $E$. coli suspension was inoculated to fresh LB medium at the ratio of $1: 100(\mathrm{v}: \mathrm{v})$ and the culture was incubated at $37^{\circ} \mathrm{C}$ with horizontal shaking $(200 \mathrm{rpm})$ for $16 \mathrm{~h}$. The culture was then added IPTG at the concentration of 1.0 $\mathrm{mmol}$, incubated for a further $5.0 \mathrm{~h}$ and the bacteria cells were harvested by centrifugation $\left(15,000 \times \mathrm{g}, 4^{\circ} \mathrm{C}, 10\right.$ $\mathrm{min})$. Subsample $(500 \mathrm{mg})$ of the isolated bacterial pellet was then suspended into $5.0 \mathrm{ml}$ of $\mathrm{PB}$ and lysozyme (Sigma, $10 \mathrm{mg} \mathrm{mL}^{-1}$ ) was added to the final concentration of $1.0 \mathrm{mg} \mathrm{mL}^{-1}$. The mixture was placed on ice for $2.0 \mathrm{~h}$, followed by mixing with TritonX-100 in the ratio of $1: 9(\mathrm{~V}: \mathrm{V})$ and subsequently centrifuged $\left(15,000 \times g, 4^{\circ} \mathrm{C}, 5 \mathrm{~min}\right)$. The resultant supernatant was analyzed by SDS-PAGE with a Bay Gene BG-Power 600 electrophoresis apparatus (Crystal Biological 
Technology Co., Ltd, Beijing, China) and the image taken by MultiMate (Universal Hood II ,BIO-RAD,US). The molecular weight of the target ClfA-FnBPB protein was $51 \mathrm{KD}$, which was purified using $\mathrm{Ni}^{+}$column using procedure described by Fan (2011). The purified ClfA FnBPB was determined for protein content as described above and was divided into two portions and stored at $80^{\circ} \mathrm{C}$ prior to use.

\subsection{Construction of Complete Antigen}

The CP8 (78 mg) obtained from a strain as described above was firstly dissolved into $15 \mathrm{~mL}$ of $\mathrm{PB}$, followed by addition of $\mathrm{ADH}$ to the final concentration of 0.5 $\mathrm{mol} / \mathrm{l}$. The solution, after adjusting $\mathrm{pH}$ to $6.5-7.2$ with concentrate $\mathrm{HCl}$ was dialyzed (MW cutoff 8,000 12,000 Dalton; Shanghai Yuanye Biological Technology Co., Ltd) against $1,000 \mathrm{ml}$ of $0.2 \mathrm{M} \mathrm{NaCl}$ solution for $2 \mathrm{~d}$, with the dialyzing solution being changed every $12 \mathrm{~h}$. At the end of the dialysis, the content in the dialysis bag that contained ADH modified CP8 was mixed with one portion of ClfA-FnBPB prepared above in the ratio (w:w) of 1:1, 2:1 or 3:1 (CP8:ClfA-FnBPB) and the EDC and sulfo-NHS were added to the final concentrations of $0.1 \mathrm{M}$ and $8.0 \mathrm{mM}$ respectively. The solution after adjusted to $\mathrm{pH}$ of 6.5-7.2 was dialyzed again against 0.2 $\mathrm{M} \mathrm{NaCl}$ solution at $4^{\circ} \mathrm{C}$ overnight. The CP8-protein conjugates (conjugation of CP8-ClfA-FnBPB) in the dialyzed solution was then purified using method described by Yang (2009). The purified CP8- ClfAFnBPB product was stored at $-80^{\circ} \mathrm{C}$ before use.

\subsection{Determination of Immunologenicity of CP8, ClfA-FnBPB and CP8-ClfA-FnBPB Antigens}

Forty Balb/c mice (SPF, 8-wk old) were randomly divided into 4 groups, which were then randomly allocated to one of the following treatments: $0.9 \% \mathrm{NaCl}$ (Control), CP8, ClfA-FnBPB and CP-ClfA-FnBPB. The mice were group-fed in an environmentally controlled room with a constant temperature of $22^{\circ} \mathrm{C}$ and $12 \mathrm{~h} / 24 \mathrm{~h}$ light. All mice were fed same pellet diet that was formulated to meet or exceed the nutrient requirements of the mice for ad libitum intake and had free access to water. After 7-d adaption, the mice in relevant group received the first immunization through intraperitoneal injection of $0.2 \mathrm{ml}$ of freshly prepared $0.9 \% \mathrm{NaCl}, \mathrm{CP} 8$, ClfA-FnBPB or CP-ClfA-FnBPB solution. The CP8 was prepared by dissolving $\mathrm{CP} 8$ into $0.9 \% \mathrm{NaCl}$ solution to designated concentration and ClfA-FnBPB and CPClfA-FnBPB solutions were prepared by diluted with $0.9 \% \mathrm{NaCl}$ solution which was then mixed with equal volume of Freund's Complete Adjuvant solution. The final concentrations of CP8, ClfA-FnBPB and CP- ClfAFnBPB in respective solutions were 50, 25 and $75 \mu \mathrm{g}$ $\mathrm{mL}^{-1}$. The second immunization was done on $\mathrm{d} 14(\mathrm{~d} 21$ of the experiment) after the first immunization using the same procedure with Freund's Inomplete Adjuvant solution as that for the first immunization. Blood samples were collected into tubes sterilized from each mouse on $\mathrm{d} 0, \mathrm{~d} 14, \mathrm{~d} 21$ and $\mathrm{d} 28$ of the experiment through amputation of tail. The blood samples were kept at room temperature for $20 \mathrm{~min}$ to obtain serum and the antibodies to the CP, ClfA-FnBPB and CP-ClfA-FnBPB in the serum of relevant treatment was determined by indirect enzyme-linked immunosorbent assay (ELISA) with spectrophotometer (TU-1800PC, PERSEE, Beijing) using procedure described by Yang (2009).

\subsection{In Vivo Challenge Testing}

At $\mathrm{d} 28$ of the experiment $\left(7\right.$ days after $2^{\text {nd }}$ immunization), the 40 mice were intraperitoneally injected $\left(0.2 \mathrm{~mL}\right.$ per mouse) with a S.aureus $\left(\mathrm{CP}^{+}\right)$cell suspension using procedure described by Wang and $\mathrm{Wu}$ (2010). The homologous S.aureus $\left(\mathrm{CP}^{+}\right)$was first aerobically grown in LB broth medium for two subcultures 18 24h each and harvested through centrifugation $(6,000 \times \mathrm{g}, 15 \mathrm{~min})$. Bacteria cell pellet was subsequently washed with $5.0 \mathrm{~mL}$ of $\mathrm{PB}$ once and the bacterial injection solution was prepared by suspending the bacterial pellet into PB to the cell density of about $1 \times 10^{9}$ colony forming unit. Immediately after injection, the mice were observed for mortality daily over the next 14 days. The dead mouse was removed from the treatment and S.aureus were isolated and identified using method of Guo et al. (2011). The live mice were killed by spinal dislocation on $\mathrm{d} 14$ after bacteria injection and the bacteria were isolated and identified as described above. The mortality of the mice was calculated as the percentage of dead mice in the total injected mice for each treatment over the 2 -wk period.

All protocols of this study, treatment and disposal of the experimental animal were reviewed and approved by the Department of Veterinary science, Inner Mongolia Agricultural University.

\subsection{Data Analysis}

The data of immunologenicity assay and challenge test were statistically analyzed using IBM SPSS Statistics 19. Data obtained from two days of sampling in immunologenicity assay were analyzed separately using analysis of variance with antigens as main factors and 
individual animal as statistical unit and data for survivability of mice in challenge test were analyzed by Chi-Square test Difference among treatment was determined by least square mean and declared at $\mathrm{p}<0.05$.

\section{RESULTS}

\subsection{Construction of CP8-ClfA-FnBPB Complete Antigen}

The content and purity of CP8 in the CP crude extract were 34.43 and $79.68 \%$ respectively. The information for ClfA-FnBPB protein was shown in Fig. 1. A clear target band shown in Fig. 1a (column 2; crude extract) was corresponding to the $51 \mathrm{KD}$ sized marker protein (column $M$ in figure). This showed that target ClfA-FnBPB protein was expressed successfully and it was soluble protein because this band of protein was not observed in precipitant (Column 1 in Fig. 1a). A major single band corresponding to the marker protein appeared after the extract being purified by $\mathrm{Ni}^{+}$column (column 1 in Fig. 1b). This indicated that purification by $\mathrm{Ni}^{+}$column increased the purity of target ClfA-FnBPB. The content of ClfA-FnBPB in the final product was $0.78 \mathrm{mg} \mathrm{mL}^{-1}$ with purity of $98 \%$.

The CP8-ClfA-FnBPB complete antigen was obtained in this study by EDC with a bridge ADH. A major absorption peak in spectrophotometry was found at $210 \mathrm{~nm}$ for the constructed CP8-ClfA-FnBPB complete antigen whist this peak was observed at 206 and $217 \mathrm{~nm}$ for CP8 and ClfA-FnBPB respectively (Fig. 2). This indicated that $\mathrm{CP} 8$ was successfully conjugated with ClfA-FnBPB to form the CP8-ClfA-FnBPB complete antigen. The $\mathrm{CP} 8$ in the product was also confirmed to be conjugated to ClfA-FnBPB by phenolsulfuric acid assay (data not shown). The contents of CP8 and ClfA-FnBPB for the CP8-ClfA-FnBPB conjugate were $499 \pm 10.4$ and $262 \pm 4.8 \mu \mathrm{g} \mathrm{mL}^{-1}$ (mean $\pm \mathrm{SD}$ ) respectively, resulting in a CP8:ClfA-FnBPB being 1.89:1 for the purified CP8-ClfA-FnBPB complete antigen.

\subsection{Immunologenicity of CP8, ClfA-FnBPB and CP8- ClfA-FnBPB Antigens}

No antibody titer was found from all mice at $\mathrm{d} 0$ and d14 after the first immunization (data not shown). Antibody titer at $\mathrm{d} 21$ and d28 ( 7 and $14 \mathrm{~d}$ after the 2 nd immunization) were significant different (Fig. 3a and b). Mice immunized with CP8 had only numerical higher antibody titer than that of immunized with $0.9 \%$ of $\mathrm{NaCl}$ at both $\mathrm{d} 21$ and $\mathrm{d} 28$. In contrast, mice immunized with CP8-ClfA-FnBPB had the highest antibody titer at $\mathrm{d} 21$, which was higher $(p<0.01)$ than that of CP8 and ClfAFnBPB groups. Antibody titer in mice immunized with ClfA-FnBPB antigen was also higher $(\mathrm{p}<0.05)$ than that of $\mathrm{CP} 8$ and $0.9 \% \mathrm{NaCl}$ groups at $\mathrm{d} 21$. The same trend was also observed at $\mathrm{d} 28$. The numerical higher antibody titer in CP8 group than in Control indicated that CP8 alone had very weak immunogenicity although it plays key role in infecting animal by bacteria.

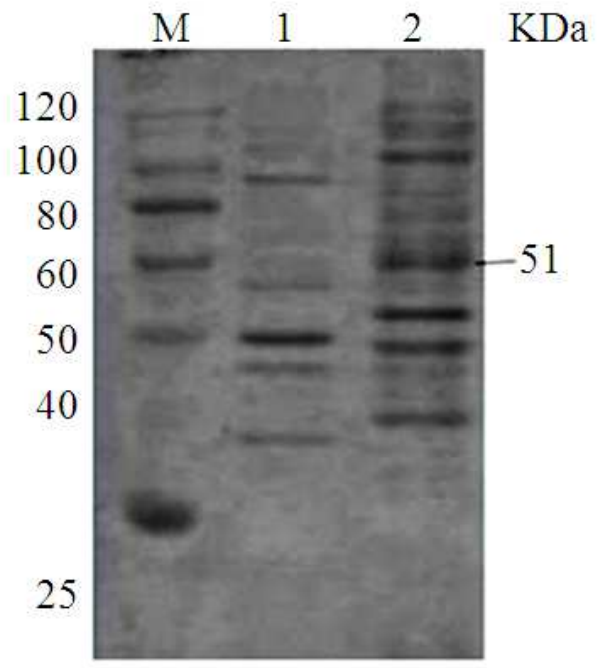

(a)

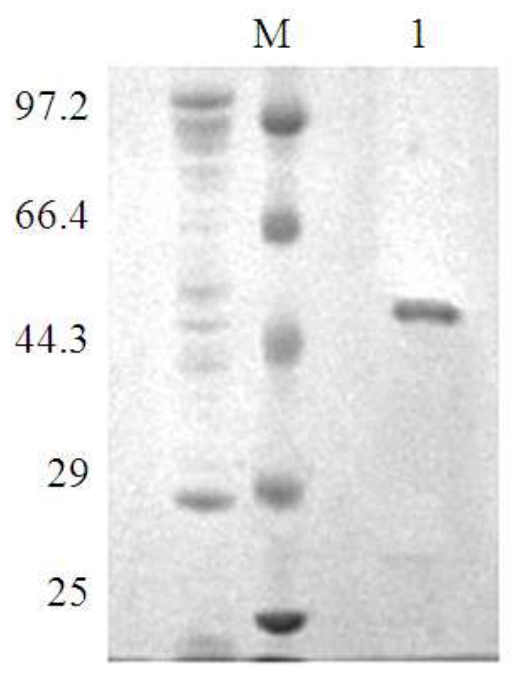

(b)

Fig. 1. Bacteria lysis analysis of pET-FnBPB-CLFA (a) and result of FnBPB-CLFA purification (b) M: Protein marker (low); A 1: pET-FnBPB-CLFA precipitate; 2: pET-FnBPB-CLFA supernatant. B 1: eluant of FnBPB-CLFA purified 
Xiuli Liu et al. / American Journal of Animal and Veterinary Sciences 8 (3): 134-141, 2013

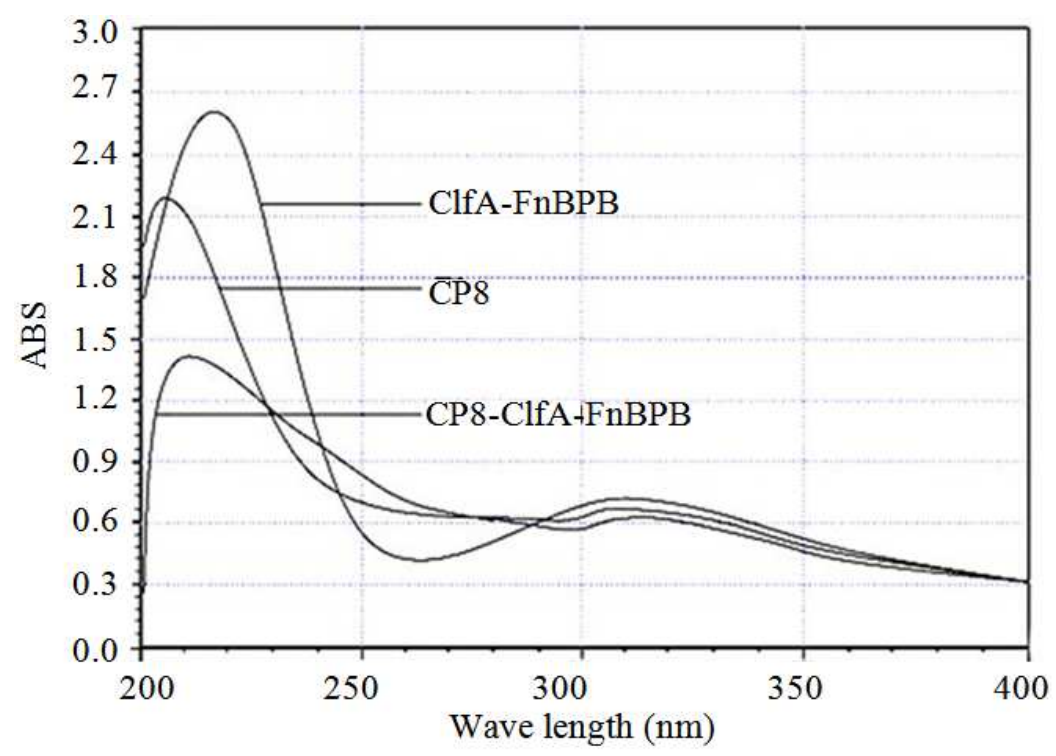

Fig. 2. Comparision of ultraviolet spectra of CP8, AlfA-FnBPB and CP8-ClfA-FnBPB

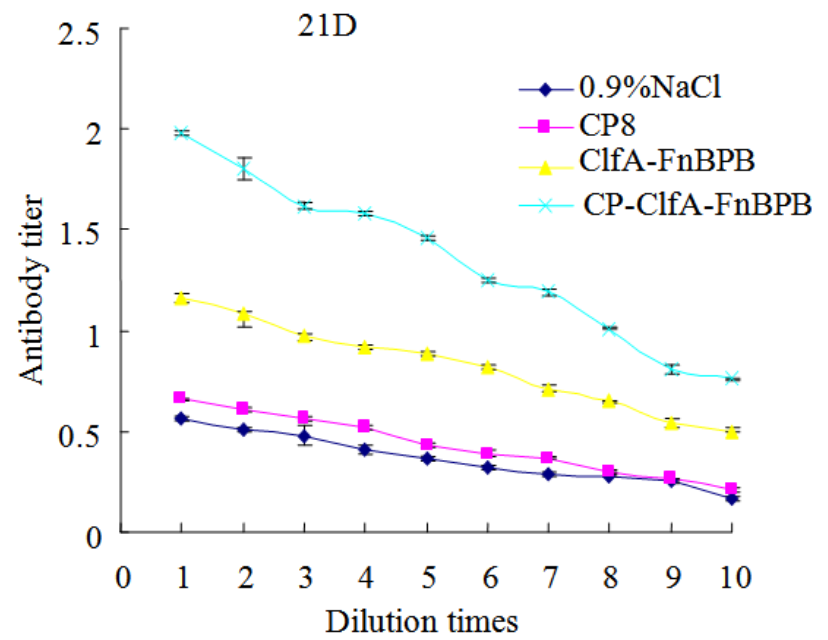

(a)

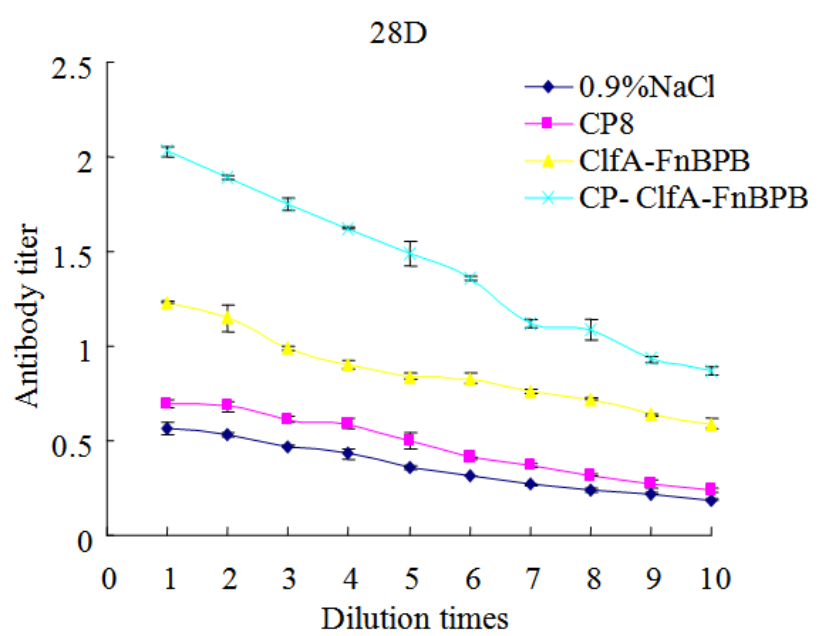

(b)

Fig. 3. Antibody titer in serum of mice immunized with $0.9 \% \mathrm{NaCl}, \mathrm{CP} 8$, ClfA-FnBPB or CP-ClfA-FnBPB on d 7 and d 14 of 2 nd immunization (d 21 and d 28 of the 1 st immunization). Dilution times were: $1,1: 50 ; 2,1: 100 ; 3,1: 200 ; 4,1: 400 ; 5,1: 800 ; 6$, $1: 1600 ; 7,1: 3200 ; 8,1: 6400 ; 9,1: 12800 ; 10,1: 25600$

Table 1. Survival rate of mice that had been immunized with $0.9 \% \mathrm{NaCl}, \mathrm{CP} 8$, ClfA-FnBPB or CP- ClfA-FnBPB for $28 \mathrm{~d}$ followed by intraperitoneally injection of CP8 serotype of S.aureus cell suspension. The number was the sum of survived mice over the two week period after bacterial infection

\begin{tabular}{llllcc}
\hline Groups & Total infected & Survived & Survival rate (\%) & Chi-square $^{\text {a }}$ & P value \\
\hline $0.9 \%$ Nacl & 10 & $2(8)^{*}$ & 20 & 13.438 & \\
CP8 & 10 & $3(7)$ & 30 & \\
ClfA-FnBPB & 10 & $6(4)$ & 60 & \\
CP8-ClfA-FnBPB & 10 & $8(2)$ & 80 & \\
\hline
\end{tabular}




\subsection{Challenge Test}

Survival rate of the mice at the end of 2-wk challenge test differed $(\mathrm{p}<0.05)$ among the four treatment groups (Table 1). Compared with $20 \%$ for Control (immunized with $0.9 \% \mathrm{NaCl}$ ), survival rate for the groups immunized with CP8, ClfA-FnBPB or CP8-ClfA-FnBPB was increased to $30,60 \quad(\mathrm{p}<0.05)$ and $80 \% \quad(\mathrm{p}<0.01)$ respectively. The survival rate of the mice immunized with CP8-ClfA-FnBPB was also higher $(\mathrm{P}<0.05)$ than that of CP8 group, higher $(\mathrm{p}<0.01)$ than $0.9 \% \mathrm{NaCl}$, higher $(\mathrm{p}<0.05)$ than ClfA-FnBPB. S. aureus were isolated from all dead mice and were confirmed by both positive mannitol test and positive coagulase test. In contrast, no $S$. aureus were isolated from any survived mice slaughtered at the end of challenge test.

The survival rate of the $S$. aureus infected mice was consistent with antibody titer of each group as described above. Therefore, the highest survival rate of mice in CP8- ClfA- FnBPB immunized group was due to the greatest immunogenicity of the CP8- ClfA-FnBPB that stimulated highest antibody titer. This demonstrated that immunization animal with CP8-ClfA-FnBPB complete antigen is much more effective than with CP8 or ClfAFnBPB alone in prevent animal from $S$. aureus infection.

\section{DISCUSSION}

Both physical and chemical methods have been used to couple CP with protein (Middleton, 2008). This study used chemical method with $\mathrm{ADH}$ as a bridge to couple $\mathrm{CP}$ with protein. The principle is that some functional groups existing in carrier can connect with half of an antigen material and $\mathrm{ADH}$ is needed as bridge to revitalize $\mathrm{CP}$ prior to making a coupling. Research has shown that coupling antigen with $\mathrm{ADH}$ produced increased immunogenicity (Liu et al., 2005; Pan, 2007) and the finding of the present research is in agreement with this. It has been shown that coupling CP with protein yielded greater antigenicity than CP alone (Woodland, 2004). Fetal bovine serum albumin, tetanus toxoid, Pseudomonas aeruginosa outside A poison, diphtheria toxin have been used as carrier protein with varying successes (Fattom et al., 2004; Mei et al., 2006; Pan, 2007; Liu et al., 2006). However, there is little information onusing ClfA-FnBPB as a carrier. This study showed that ClfA-FnBPB was successfully coagulated with CP8 forming complete antigen, indicating ClfA-FnBPB could be potentially effective protein carrier.
This is due to the fact that CP8 belongs to small molecular weight substances that usually possess weak or no immunogenicity (Katherine and Lee, 2004; Wu, 2011). Similarly, the higher antibody titer observed in ClfA-FnBPB group than that in Control and in CP8 is also likely due to the increased molecule size of the ClfA-FnBPB. The markedly higher antibody titer in CP8- ClfA-FnBPB group than in other groups indicated that coupling $\mathrm{CP} 8$ with ClfA-FnBPB significantly increased immunogenicity of ClfA-FnBPB. Because CP belonged to T-Independent Antigen (TI-Ag), connecting it conjugately with protein, would transfer it into $\mathrm{T}$ Dependent Antigen (TD-Ag) and yield enhanced immunologenicity. It has also been suggested that CP would become effective antigen when it is connected with carrier protein into conjugates $(\mathrm{Wu}, 2011)$. Other researches have showed that $\mathrm{CP}$ itself had weaker antigenicity that was unable to stimulate animal's body to produce antibody. John et al. (2011) also suggested that immunogenicity of conjugation of CPs and bacteria protein would be considerably higher than that of CPs alone.

Reasearchers (Lorena et al., 2008; Gaudreau et al., 2007; Broullette et al., 2002) also reported that immunization with protein conjugation vaccines were used efficently. CPs-ClfA conjugation was more effective than CPs and ClfA separately in preventing staphylococcal infection in a mouse model of mastitis. Further research is need to evaluate the immunogenicity of the CP8- ClfA- FnBPB in large animals.

\section{CONCLUSION}

CP8 and ClfA-FnBPB were successfully conjugated to produce CP8-ClfA-FnBPB complete antigen. Conjugation of ClfA-FnBPB with CP8 markedly increased level of antibody titre, immunogenicity of the antigen and survival rate of the infected animal as compared to CP8 or ClfA-FnBPB alone. Therefore, developing vaccine based on CP8ClfA-FnBPB complete antigen is a potential strategy to battle with bovine mastitis.

\section{ACKNOWLEDGEMENT}

We are grateful for Dr. Tessie McNeely (Merck company, U.S) for providing the gift of monoclonal antibody to our laboratory, so we can make a basic research surveys in order to further research capsular polysaccharide in China. 


\section{REFERENCES}

Broullette, E., P. Lacasse, L. Shkreta, J. Belanger and G. Grondin et al., 2002. DNA immunization against Chumping factor(CIfA) of Staphylococcus aureus. Vaccine, 17-18: 2348-2357. PMID: 12009291

Chang, B.S., J.S. Moon, H.M. Kang, Y.I. Kim and H.K. Lee et al., 2008. Protective effects of recombinant staphylococcal enterotoxin type $\mathrm{C}$ mutant vaccine against experimental bovine infection by a strain of Staphylococcus aureus isolated from subclinical mastitis in dairy cattle. Vaccine, 17: 2081-2091. DOI: $10.1016 /$ j.vaccine.2008.02.043

Creuzenet, C. and S.L. Joseph, 2001. Topological and functional characterization of $\mathrm{WbpM}$, an inner membrane UDP-GlcNAc C6 dehydratase essential for lipopolysaccharide biosynthesis in Pseudomonas aeruginosa. J. Mol. Microbiol., 41: 1295-1310. DOI: 10.1046/j.1365-2958.2001.02589.x

Daum, R.S. and B. Spellberg, 2012. Progress Toward a Staphylococcus aureus Vaccine. Clin. Infect. Dis., 54: 560-567. DOI: $10.1093 / \mathrm{cid} / \mathrm{cir} 828$

Fan, X., 2011. Research about subunit vaccine against Staphylococcus aureus induced mastitis. D. Inner Mongolia Agricultural University.

Fattom, A., S. Fuller and M. Propst, 2004. Safety and immunogenicity of a booster dose of Staphylococcus aureus types 5 and 8 capsular polysaccharide conjugate vaccine (StaphVAX) in hemodialysis patients. Vaccine, 23: 656-663. PMID: 15542186

Fournier, J.M., W.F. Vann, W.W. Karakawa, 1984. Purification and characterization of Staphylococcus aureus type 8 capsular polysaccharide. Infect. Immunity, 45: 87-93. PMID: 6429051

Gaudreau, M.C., P. Lacasse and B.G. Talbot, 2007. Protective immune responses to a multi-gene DNA vaccine against Staphylococcus aureus. Vaccine, 5: 814-824. PMID: 17027124

Guo, Y., X.L. Liu, A.R. Zhang and Y.Q. Hao, 2011. Identification of main serotype capsular polysaccharide of Staphylococcus aureus in Dairy cow mastitis. Heilongjiang Anim. Husbandry Vet.

James, C., H. Robert and P. Greg, 2009. Staphylococcus aureus capsule type 8 antibodies provide inconsistent efficacy in murine models of staphylococcal infection. J. Landes Biosci., 5: 254263. PMID: 18787395

John, B., Robbins, R. Schneerson, S.C. Szu, V. Pozsgay, 2011. Polysaccharide-protein conjugate vaccines. History Vaccine Dev.
Josefsson, E., O. Hartford, L. O'Brien, J.M. Patti and T. Foster, 2001. Protection against experimental Staphylococcus aureus arthritis by vaccination with clumping factor A, a novel virulence determinant. J. Infect. Dis., 184: 1572-1580. PMID: 11740733

Katherine, O.R. and J.C. Lee, 2004. Staphylococcus aureus capsular polysaccharides. Clin. Microbiol. Rev., 17: 218-234. PMID: 14726462

Lin, B.F., N.S. Zhang and L.H. Yuan, 2005. Research progress in Staphylococcus aureus capsule polysaccharide. J. Progress Vet. Med., 26: 44-461.

Liu, F.L., B. Wu, L. Du, M. Yang and Z.J. Wang et al., 2005. Preparation, characterization and immunogenecity in mice for conjugate vaccine of Nesseria meningococcal sero-group a polysaccharide-tetanus toxoid. Progress Microbiol. Immunol., 33: 14-21.

Liu, F.L., B. Wu, L. Du, X. Cao, M. Yang, 2006. Immunities of Nesseria Meningococcal Serogroup A polysaccharide-prorein conjugate vaccines preparation with different carrier proteins. Chin. J. Biologicals, 19: 44-48.

Lorena, P.N., R.B. Fernanda, P.A. Lucía, J.C. Lee and D.O. Sordelli, 2008. Antibodies to capsular polysaccharide and clumping factor a prevent mastitis and the emergence of unencapsulated and small-colony variants of staphylococcus aureus in mice. J. Infect. Immunity, 76: 5738-5744. PMID: 18809660

Mei, M.H., B. Bolgiano and A. Martino, 2006. Preclinical laboratory evaluation of a bivalent Staphylococcus aureus saccharide-exotoxin A protein conjugate vaccine. Hum. Vaccin., 2: 89-98. PMID: 17268204

Middleton, J.R., 2008. Staphylococcus aureus antigens and challenges in vaccine development. Expert Rev. Vaccines, 7: 805-815. DOI: 10.1586/14760584.7.6.805

Ni Eidhin, D., S. Perkins, P. Francois, P. Vaudaux and M. Hook et al., 1998. Clumping factor B (ClfB), a new surface-located fibrinogen-binding adhesin of Staphylococcus aureus. Mol. Microbiol., 30: 245257. PMID: 9791170

Pan, 2007. Research on preparation, identification and immunology of complete antigen capsular polysaccharides in Staphylococcus aureus. M. Jilin. Jilin University.

Reid, A.N. and C.M. Szymanski, 2010. Chapter 20Biosynthesis and assembly of capsular polysaccharides. Microbial. Glycobiol. Struct. Relevance Applic. DOI: 10.1016/B978-0-12374546-0.00020-1 
Siqinmenghe, L.B., 2012. Research on sustainable development of the dairy industry of Inner Mongolia-take suburb dairy farmers in Hohhot, Baotou for example. Northern Econ.

Stutz, K., R. Stephan and T. Tasara, 2011. SpA, ClfA, and FnbA genetic variations lead to Staphaurex testnegative phenotypes in bovine mastitis Staphylococcus aureus isolates. J. Clin. Microbiol., 49: 638-646. DOI: 10.1128/JCM.01148-10

Visai, L., N. Yanagisawa, E. Josefsson, A. Tarkowski and I. Pezzali et al., 2009. Immune evasion by Staphylococcus aureus conferred by iron-regulated surface determinant protein IsdH. Microbiology, 155: 667-679. PMID: 19246738

Wang, W.Y. and D.F. Wu, 2010. Research about enhancing immuneresponses technicals of CP5 and CP8 in Staphylococcus aureus. Fujian Agriculture University, Fujian, China.

Wang, X., 2008. The combination chemistry and test methods of conjugate vaccine-polysaccharide and protein. Chin. J. Biol.

Wann, E.R., S. Gurusiddappa and M. Hook, 2000. The fibronectin-binding MSCRAMM FnbpA of Staphylococcus aureus is a bifunctional protein that also binds to fibrinogen. J. Biol. Chem., 275: 1386313871. PMID: 10788510
Woodland, D.L., 2004. Jump-starting the immune system: primeboosting comes of age. Trends Immunol., 25: 98-104. PMID: 15102369

Wu, J.Y., 2011. Preparations and immunogenicity analysis of conjugated antigens composed of the Staphylococcus aureus surface. M. Shihezi Univercity, Shihezi.

Wu, Y.X. and L.Q. Huang, 2003. The immunology and pharmacology mechanism of the prevention and control of dairy cow mastitis. J. Chinese J. Vet. Med., 39: 40-44. DOI: 10.3969/j.issn.05296005.2003.03.019

Yang, Q., 2009. Preparation of monoclonal antibody and the establishment of the Elisa method about type 5 capsular polysaccharides in Staphylococcus aureus. Jilin University, M. Jilin.

Zhang, R.F. and X.D. Wang, 2003. Research progress in Staphylococcus aureus vaccine. J. Fascicule Book Foreign Med. Microbiol., 26: 23-25. DOI: 10.3969/j.issn.1673-6184.2003.04.010

Zhang, Y., H.Z. Wu. and H.B. Yi, 2004. Development and preliminary application of typing serum of Capsule Polysaccharide in Staphylococcus aureus. J. Progress Microbiol. Immunol., 32: 7-91. 